\title{
High CFP score indicates poor prognosis and chemoradiotherapy response in LARC patients
}

\author{
Siyi Lu ${ }^{1 \dagger}$, Zhenzhen Liu ${ }^{1 \dagger}$, Bingyan Wang ${ }^{1}$, Fei Li ${ }^{1}$, Yan Meng ${ }^{1}$, Junwei Wang ${ }^{1}$, Yuxia Wang ${ }^{2}$, Hao Wang ${ }^{2 *}$, \\ Xin Zhou ${ }^{1 *}$ and Wei Fu ${ }^{1 *}$ (1)
}

\begin{abstract}
Background: Preoperative tumor markers, inflammation, and nutritional status are considered important predictors of prognosis and tumor response in locally advanced rectal cancer (LARC) patients. This study aims to explore the prognostic and predictive role of carcinoembryonic antigen (CEA), the Fibrinogen-Albumin Ratio Index (FARI), the Prognostic Nutritional Index (PNI) in LARC patients and compared them with a novel combined CEA-FARI-PNI (CFP) scoring system.
\end{abstract}

Methods: A total of 138 LARC patients undergoing radical surgery following neoadjuvant chemoradiotherapy (NCRT) between January 2012 and March 2019 were enrolled. The X-tile program was used to determine the optimal cut-off values of CEA, FARI, and PNI, and CFP scoring system was constructed accordingly. The prognostic ability of these factors was assessed by the time-dependent receiver operating characteristic (ROC) curve, Kaplan-Meier, Cox regression, and logistic regression. Nomogram was established to evaluate the predictive role of these factors in tumor response.

Results: The optimal cut-off values of CEA, FARI, and PNI were $5.15 \mathrm{ng} / \mathrm{l}, 10.56 \%$, and $42.25 \mathrm{~g} / \mathrm{L}$, respectively. The timedependent ROC curve showed that compared to CEA, FARI, and PNI, CFP showed stable predictive efficacy for overall survival (OS) and disease-free survival (DFS). In multivariate analysis, CFP was the only factor that could independently predict OS (HR=8.117, $p=0.001)$ and DFS ( $H R=4.994, p<0.001)$. Moreover, high CFP $(O R=3.693, p=0.002)$ was also an independent risk factor of poor response. The area under the ROC curve (AUC) of the nomograms for predicting tumor response was better including CFP $(0.717)$ than without CFP $(0.656)(p<0.05)$.

Conclusions: The CFP score was a more reliable marker for predicting OS, DFS, and NCRT efficacy in LARC patients, and the score could apparently improve predicted efficacy of the nomogram.

Keywords: Rectal cancer, CFP, Prognosis, Tumor regression grade

\footnotetext{
*Correspondence: wanghaobysy@bjmu.edu.cn; zhouxinasd@sina.cn; fuwei@bjmu.edu.cn

${ }^{\dagger}$ Siyi Lu and Zhenzhen Liu contributed equally to this work ${ }^{1}$ Department of General Surgery, Peking University Third Hospital, Beijing 100191, China

2 Department of Radiotherapy, Peking University Third Hospital, Beijing 100191, China
}

\begin{abstract}
Introduction
Colorectal cancer is one of the most common cancers worldwide and is the second leading cause of cancerrelated deaths [1]. Rectal cancer accounts for nearly $30 \%$ of all colorectal cancers [2]. Currently, preoperative neoadjuvant chemoradiotherapy (NCRT) is thought to improve local pelvic control and decrease the incidence of local relapse and has become the standard regimen
\end{abstract}

(c) The Author(s) 2021. This article is licensed under a Creative Commons Attribution 4.0 International License, which permits use, sharing, adaptation, distribution and reproduction in any medium or format, as long as you give appropriate credit to the original author(s) and the source, provide a link to the Creative Commons licence, and indicate if changes were made. The images or other third party material in this article are included in the article's Creative Commons licence, unless indicated otherwise in a credit line to the material. If material is not included in the article's Creative Commons licence and your intended use is not permitted by statutory regulation or exceeds the permitted use, you will need to obtain permission directly from the copyright holder. To view a copy of this licence, visit http://creativeco mmons.org/licenses/by/4.0/. The Creative Commons Public Domain Dedication waiver (http://creativecommons.org/publicdomain/ zero/1.0/) applies to the data made available in this article, unless otherwise stated in a credit line to the data. 
for locally advanced rectal cancer (LARC) patients. Approximately $50-60 \%$ of patients are downstaged after NCRT, and 10-30\% will achieve a pathological complete response [3]. Although standard treatments are available for these patients, including NCRT, total mesorectal excision (TME), and adjuvant chemotherapy, local relapse and distant metastasis remain the leading problems of LARC $[4,5]$. Hence, more economical and feasible preoperative clinical biomarkers are needed to stratify patients with high-risk status and to guide tailored treatment.

Carcinoembryonic antigen (CEA) is widely used as a prognostic marker for colorectal cancer patients worldwide. Previous studies [6-8] have shown that serum CEA was associated with tumor response and prognosis in rectal cancer patients undergoing curative excision. Moreover, the preoperative CEA level may play a determinant role in the early detection of recurrent disease during follow-up after the TME procedure.

The cancer-related systemic inflammatory response and alterations in nutritional status have been identified as some of the most critical hallmarks of solid tumors $[9,10]$. Inflammation may facilitate the proliferation and distance seeding of malignant cells, leading to tumor progression and metastasis, inhibiting adaptive immunity, and even altering tumor sensitivity to NCRT [11-13]. Meanwhile, malnutrition is associated with decreased immune function [14], weakened physical status[15], and poor NCRT outcomes [16], leading to increased mortality among cancer patients. The fibrinogen-to-albumin index (FARI) is considered an essential biomarker that reflects both systemic inflammatory status and nutritional status, and several studies have reported that FARI is closely related to the prognosis of various cancers, such as breast cancer [17], esophageal cancer [18], and gastric cancer [19]. Our previous findings have shown similar results in LARC patients undergoing TME following NCRT, and we have found that FARI is associated with tumor response [20]. The prognostic nutritional index (PNI), based on the albumin level and lymphocyte count, is another widely used biomarker that combines inflammatory and nutritional parameters. Okugawa et al. [21] analyzed 114 rectal cancer patients who underwent NCRT and demonstrated that PNI could predict survival and tumor response.

Since CEA [7], FARI [20] and PNI [21] have all been found to serve as indicators of the prognosis and tumor response of LARC patients, we constructed a combination of these markers and investigated the prognostic and predictive role of the combination (CEA-FARI-PNI, CFP) in 138 LARC patients undergoing radical surgery following NCRT and compared its prognosis predicted efficacy with CEA, FARI and PNI. Here, we reported a novel CFP scoring system could independently predict survival of
LARC patients and precisely identify different NCRT response among LARC patients.

\section{Methods \\ Study population}

A total of 138 consecutive LARC (cTNM stage II or stage III) patients from Peking University Third Hospital between March 2012 and March 2019 were ultimately enrolled and followed. Ethical approval was obtained from the Ethics Committee of Peking University Third Hospital (IRB00006761-M2019387), and this study adhered to the tenets of the Declaration of Helsinki. The inclusion criteria were as follows: (1) diagnosis of LARC through preoperative MR and CT and received NCRT followed by radical surgery; (2) diagnosis of adenocarcinoma via postoperative histopathologically; (3) complete resection without positive tumor margins; and (4) complete inpatient data, including preoperative complete blood counts and follow-up data. The exclusion criteria were as follows: (1) anti-immunosuppressive or anti-inflammatory treatments; (2) autoimmune disease, hematological disease, and acute infection; 3) the presence of other cancers in addition to rectal adenocarcinoma; and 4) emergency surgery for obstruction or perforation of the rectum.

\section{Clinicopathological data and definitions}

Hematological examinations included routine blood examination, liver function tests, coagulation tests, and CEA measurements. All blood specimens were tested in the laboratory of our hospital within two weeks before the operation. PNI and FARI were defined as follows: $\mathrm{PNI}=$ albumin $(\mathrm{g} / \mathrm{L})+5 \times$ lymphocyte count $\left(10^{9} / \mathrm{L}\right) ; \mathrm{FARI}=$ the ratio of fibrinogen $(\mathrm{g} / \mathrm{L})$ to albumin $(\mathrm{g} / \mathrm{L}) \times 100 \%$. The AJCC-TRG definitions were as follows: TRG0, no sign of tumor cells; TRG1, single tumor cell or small groups of tumor cells can be detected; TRG2, residual cancer with a desmoplastic response (mild regression); and TRG3, no regression. In this study, TRG0-1 was defined as a good response, while TRG2-3 was defined as a poor response.

\section{Treatment and follow-up}

All eligible patients received radiation according to institutional protocols. Oral capecitabine at a dose of $1,650 \mathrm{mg} / \mathrm{m}^{2}$ per daily was administered concurrently with radiotherapy. Six to 9 weeks after the end of chemoradiotherapy, the LARC patients underwent curative TME, which was performed by four experienced colorectal surgeons at Peking University Third Hospital. Patients were followed-up at 1 and 3 months after surgery and every 6 months thereafter. Abdominal and pelvic contrast-enhanced CT or MRI scans and CEA levels were 
routinely performed every 6 months for 2 years and then once every year for a total of 3 years at each follow-up. Colonoscopy was conducted within 1 year after surgery and then repeated every 2-3 years. The presence of new lesions revealed by biopsy or imaging was deemed tumor recurrence. Appropriate treatment, such as repeated surgery, systemic chemotherapy, radiofrequency ablation, or RT, was performed for patients with tumor recurrence. The period from radical surgery to death was defined as OS, and the period from radical surgery to any local or distant recurrence was defined as DFS.

\section{Construction of the novel prognostic scoring system}

A novel tumor marker, inflammation- and nutritionbased prognostic score, CFP (a combination of CEA, FARI, and PNI), was constructed in this study. CEA levels and FARI scores lower or higher than the cut-off values were considered 0 and 1 point, respectively, while levels of PNI higher or lower than the cut-off values were considered 0 and 1 point, respectively. Total scores of 0 and $\geq 1$ were defined as low and high CFP scores, respectively (Fig. 1).

\section{Statistical analysis}

The X-tile program was used to determine the optimal cut-off values of CEA, FARI, and PNI. The timedependent ROC analysis to compare the prognostic values of the markers for DFS and OS was performed by 'timeROC' packages in $\mathrm{R}$ version 3.5.2. Normality was tested using the Shapiro-Wilk test. Independent sample t-tests, chi-square tests, and Fisher's exact tests were used to analyze the correlation between the CFP score and clinicopathological parameters. KaplanMeier curves of patients stratified by CEA, FARI, PNI, and CFP values were generated for DFS and OS, and the log-rank test was used to calculate p values. Univariate and multivariate analyses of the Cox proportional hazards model were used to determine the factors that may correlate with DFS and OS, while univariate and multivariate analyses of logistic regression were used to determine the factors that may be associated with TRG. Potential risk factors $(\mathrm{P}<0.1)$ were adopted for multivariate analysis with the backward stepwise method following univariate analysis. According to the multivariate analysis results of logistic regression, a prognostic nomogram for predicting the TRG of LARC patients was established, and the AUC and calibration curve verified its predictive ability. The logistic regression nomogram was established by the 'rms' package in R. All statistical analyses were carried out by SPSS Statistics 19.0 (IBM Corporation, Armonk, NY, USA). A P value $<0.05$ was recognized as statistically significant.

\section{Results}

\section{Patient characteristics}

Among the 138 LARC patients enrolled (Additional file 1: Figure S1), male patients accounted for the majority (72.5\%), and the median age was 60 years (range 53-69). A total of 118 (85.5\%) patients had tumors located in the mid-low rectum, and 63 (45.7\%) patients had a tumor size $>5 \mathrm{~cm}$. Seventy-four $(53.6 \%)$ patients were downstaged to stage 0-I after NCRT, while 64 patients remained in stage II-III. Eight (5.8\%), $17(12.3 \%)$ and $20(14.5 \%)$ patients had positive lymphovascular invasion (LVI), perineural invasion, and tumor deposits, respectively. According to the fourtier AJCC-TRG, 80 (58\%) were TRG0-1, while 58 (42\%) were TRG2-3. The median CEA, FARI, and PNI values were 3.5 (range 1.8-4.1), 7.7\% (range 6.5-8.7), and 45.9 (range 43.2-48.5), respectively. Detailed characteristics of the enrolled patients are shown in Table 1.

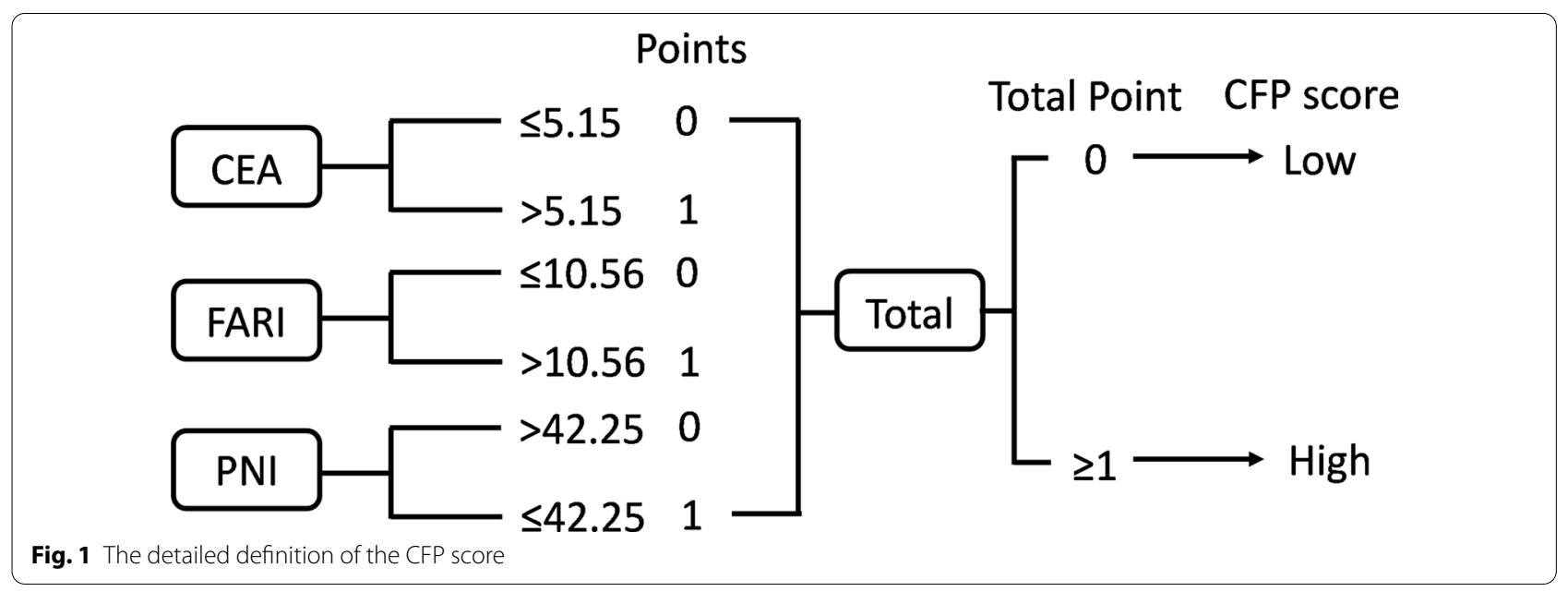


Table 1 Patient characteristics

\begin{tabular}{|c|c|}
\hline Variables & Total number (\%) \\
\hline \multicolumn{2}{|l|}{ Gender } \\
\hline Male & $100(72.5)$ \\
\hline Female & $38(27.5)$ \\
\hline Age, years [median (IQR)] & $60(53-69)$ \\
\hline \multicolumn{2}{|l|}{ Site } \\
\hline Low & $43(31.2)$ \\
\hline Middle & $75(54.3)$ \\
\hline High & $20(14.5)$ \\
\hline \multicolumn{2}{|l|}{ Tumor size } \\
\hline$>5 \mathrm{~cm}$ & $63(45.7)$ \\
\hline$\leq 5 \mathrm{~cm}$ & $75(54.3)$ \\
\hline \multicolumn{2}{|l|}{ CTNM } \\
\hline$\|$ & $31(22.5)$ \\
\hline III & $107(77.5)$ \\
\hline \multicolumn{2}{|l|}{ ypTNM } \\
\hline $0-1$ & 74 (53.6) \\
\hline$\|-\| \|$ & $64(46.4)$ \\
\hline \multicolumn{2}{|l|}{ Histopathology } \\
\hline Well differentiation & $6(4.7)$ \\
\hline Moderate differentiation & $109(84.5)$ \\
\hline Poor differentiation & $14(10.9)$ \\
\hline \multicolumn{2}{|l|}{ LVI } \\
\hline Positive & $8(5.8)$ \\
\hline Negative & $130(94.2)$ \\
\hline \multicolumn{2}{|l|}{ PNI } \\
\hline Positve & $17(12.3)$ \\
\hline Negative & $121(87.7)$ \\
\hline \multicolumn{2}{|l|}{ Tumor deposits } \\
\hline Positive & $20(14.5)$ \\
\hline Negative & $118(85.5)$ \\
\hline \multicolumn{2}{|l|}{ TRG } \\
\hline $0-1$ & $80(58.0)$ \\
\hline $2-3$ & $58(42.0)$ \\
\hline LNH [median (IQR)] & $8.8(5.0-12.0)$ \\
\hline CEA [median (IQR)] & $3.5(1.8-4.1)$ \\
\hline FARI\% [median (IQR)] & $7.7(6.5-8.7)$ \\
\hline PNI [median (IQR)] & $45.9(43.2-48.5)$ \\
\hline \multicolumn{2}{|l|}{ CFP } \\
\hline Low & $95(68.8)$ \\
\hline High & $43(31.2)$ \\
\hline
\end{tabular}

$I Q R$ interquartile rangel, $L V I$ lymphovascular invasion, $P N I$ perineural invasion, TRG tumor regression grade, $L H N$ lymph node harvest, CEA carcinoembryonic antigen, $P N I$ prognostic nutrition index, FARI fibrinogen-Albumin Ratio Index, CFP CEA-FARI-PNI score

\section{Optimal cut-off values of CEA, FARI and PNI}

According to the X-tile program, the optimal cut-off values of OS in CEA, FARI, and PNI were $5.15 \mathrm{ng} / \mathrm{ml}$, $10.56 \%$, and $42.25 \mathrm{~g} / \mathrm{L}$, respectively. Detailed data are shown in Fig. 2. Based on these cut-off values, patients were divided into low CEA $(\leq 5.15, \mathrm{n}=122)$, FARI $(\leq 10.56, \mathrm{n}=124)$, PNI $(\leq 42.25, \mathrm{n}=27)$ and high CEA $(>5.15, \mathrm{n}=16)$, FARI $(>10.56, \mathrm{n}=14)$, and PNI $(>42.25$, $\mathrm{n}=111$ ) groups. The CFP scores of CEA, FARI, and PNI were obtained based on the cut-off values of the X-tile program. Likewise, the low $(n=95)$ and high $(n=43)$ CFP score groups were also constructed according to the final CEA + FARI + PNI scores.

\section{Time-dependent ROC analysis of CEA, FARI, PNI, and CFP}

Time-dependent ROC analysis was conducted to compare the ability of the markers to predict OS and DFS. From the second year after surgery, the AUC of CFP for forecasting OS continued to be superior to those of CEA and FARI (Fig. 3a). Meanwhile, the AUC of CFP in forecasting DFS was superior to those of CEA and PNI (Fig. 3b). Unlike CEA, FARI, and PNI, CFP showed a relatively stable ability to predict both OS and DFS. The AUCs of the CFP in predicting 1, 2, 3, 4, 5, and 6 years for OS and DFS were 0.846, 0.847, 0.768, 0.777, 0.75, and 0.682 and $0.754,0.704,0.739,0.77,0.749$, and 0.671 , respectively. The data for CEA, FARI, and PNI are shown in Additional file 1: Table S1.

\section{The correlation between CFP and clinicopathological characteristics}

We next analyzed the relationship between CFP and clinicopathological characteristics in LARC patients. The chi-square test showed that the high CFP score group was significantly associate with larger tumor size $(\mathrm{p}=0.002)$, higher ypTNM stage $(<0.001)$, the presence of perineural invasion $(\mathrm{p}<0.001)$, and poorer tumor response $(\mathrm{p}=0.001)$ compared to the low CFP score group. The CFP score was not significantly correlated with the remaining clinicopathological features, such as sex, age, tumor site, histopathology, total number of lymph nodes harvested (LNH), LVI, and tumor deposits $(p>0.05)$. The detailed data of the two groups are shown in Table 2.

\section{Survival analysis of CEA, FARI, PNI, and CFP in LARC}

The follow-up time ranged from 5 to 100 months, and the median follow-up time was 48.5 months. Fifteen (10.9\%) patients had died at the last follow-up, and local recurrence with or without metastasis occurred in 24 (17.4\%) patients among the 138 eligible patients. According to Kaplan-Meier analysis of OS, CEA (0.0083), FARI $(\mathrm{p}<0.0001)$, PNI $(\mathrm{p}<0.0001)$ and CFP $(\mathrm{p}=0.0001)$ could distinguish patients with poor OS (Fig. $4 \mathrm{a}, \mathrm{c}, \mathrm{e}, \mathrm{g}$ ), and the cumulative 5-year OS rates of high CEA, high FARI, low PNI, and high CFP were $67.7 \%, 60.2 \%, 59.1 \%$ and $71.5 \%$, respectively. high FARI $(<0.0001)$, low PNI $(\mathrm{p}=0.0003)$ 

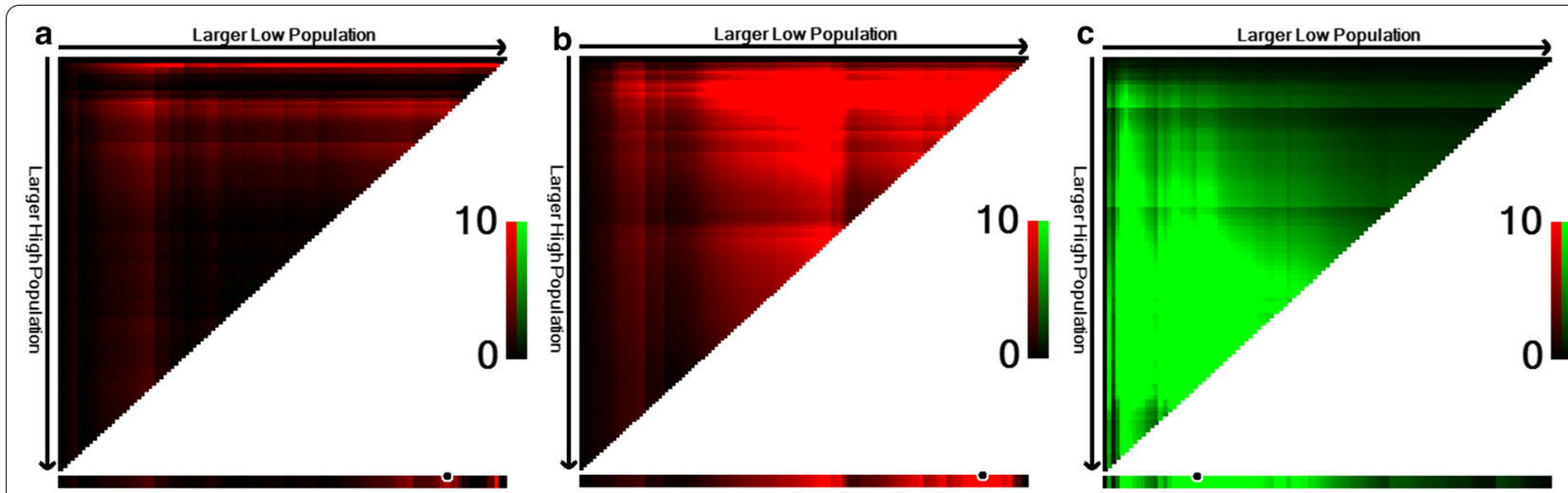

Fig. 2 X-tile plot of CEA (a), FARI (b), and PNI (c). The x-axis of the X-tile plot represented all possible cut-off values for the marker, with the size of the cut-off values increasing from left to right. The brightest pixel (marked by the black circle) represented the optimal cut-off value. Red colouration indicated an indirect association between markers and prognosis, in which green colouration indicated a direct association between markers and prognosis
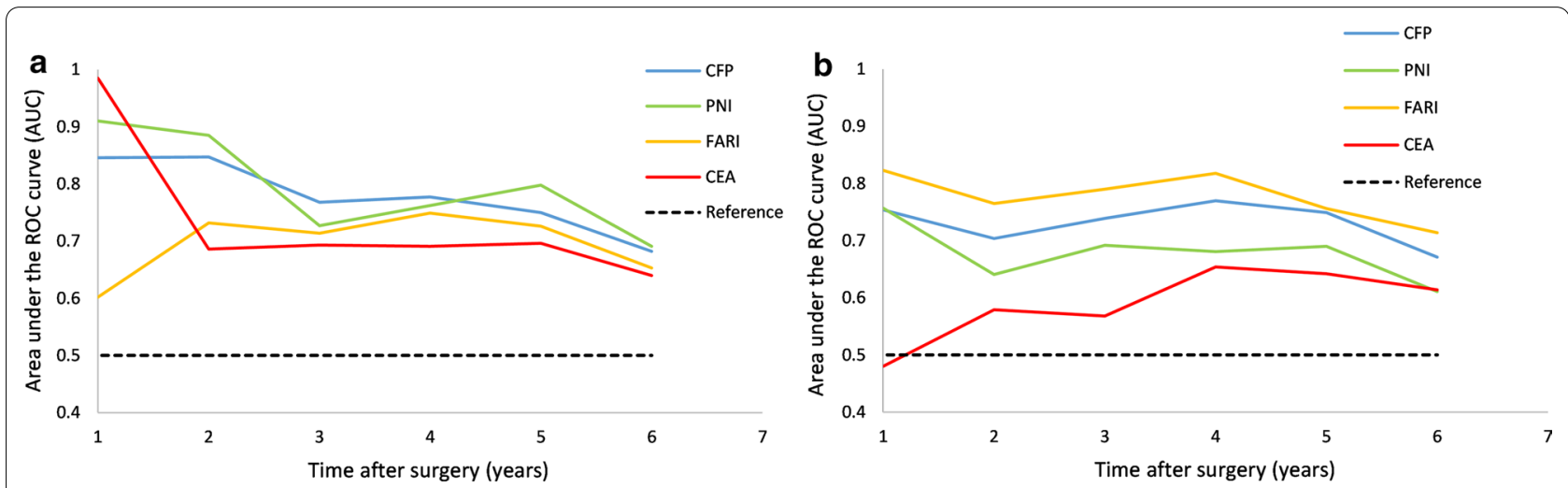

Fig. 3 Time-dependent ROC curve. Time-dependent ROC of preoperative CEA, PNI, FARI, and CFP for OS (a) and DFS (b)

and high CFP $(<0.0001)$ were significantly correlated with poor DFS (Fig. 4b, d, f, h), and the cumulative 5-year DFS rates of high FARI, low PNI, and high CFP were $30.6 \%, 49.0 \%$, and $54.7 \%$, respectively.

\section{Univariate and multivariate analysis for OS and DFS}

A Cox proportional hazard model was conducted further to demonstrate the prognostic value of the CFP scoring system. Univariate analysis showed that ypTNM stage, the presence of LVI, perineural invasion, tumor deposits, CEA, FARI, PNI, and CFP were significantly associated with OS (Table 3). All potential risk factors $(\mathrm{p}<0.1)$ were adopted for multivariate analysis, and results showed that both a high CFP score $(H R=6.606, p=0.005)$ and the presence of LVI $(\mathrm{HR}=7.019, \mathrm{p}=0.001)$ were independent prognostic factors of poor OS in LARC patients undergoing radical surgery following NCRT. As for DFS, univariate analysis showed that tumor size, ypTNM stage, the presence of LVI, perineural invasion, and tumor deposits, FARI, PNI, and CFP were significantly associated with it (Table 3). Multivariate analysis showed that both CFP score $(\mathrm{HR}=6.635, \mathrm{p}=0.003)$, ypTNM stage $(\mathrm{HR}=4.785, \mathrm{p}=0.02)$, perineural invasion $(\mathrm{HR}=4.904$, $\mathrm{p}=0.009)$, tumor deposits $(\mathrm{HR}=7.932, \mathrm{p}<0.001)$, and FARI $(H R=3.642, p=0.013)$ were independent prognostic indicator of DFS in LARC patients undergoing radical surgery following NCRT. (Table 3).

\section{The relationship between CEA, FARI, PNI, and CFP and response to NCRT}

To further explore the clinical utility of CEA, FARI, PNI, and CFP in predicting tumor response to NCRT, ROC curves and logistic regression models were established based on TRG. According to the ROC analysis, the AUC of CFP to predict TRG was $0.633(\mathrm{p}=0.008)$, which was superior to those of CEA (AUC $=0.549, \mathrm{p}=0.330$ ), FARI (AUC $=0.517, \mathrm{p}=0.740)$, and PNI $(\mathrm{AUC}=0.584$, $\mathrm{p}=0.093$ ) (Fig. 5a). In the forest plot of univariate 
Table2 Characteristics of patients according to CFP score

\begin{tabular}{|c|c|c|c|}
\hline Variables & Low CFP group & High CFP group & $\mathrm{p}$ value \\
\hline Gender & & & 0.729 \\
\hline Male & $68(71.6)$ & $32(74.4)$ & \\
\hline Female & $27(28.4)$ & $11(25.6)$ & \\
\hline Age, years [median $(\mathrm{Cl})]$ & $60(58-63)$ & $60(56-64)$ & 0.899 \\
\hline Site & & & 0.076 \\
\hline Low & $35(36.8)$ & $8(18.6)$ & \\
\hline Middle & $46(48.4)$ & $28(67.4)$ & \\
\hline Upper & $14(14.7)$ & $6(14.0)$ & \\
\hline Tumor size & & & 0.002 \\
\hline$>5 \mathrm{~cm}$ & $35(36.8)$ & $28(65.1)$ & \\
\hline$\leq 5 \mathrm{~cm}$ & $60(63.2)$ & $15(34.9)$ & \\
\hline ypTNM category & & & $<0.001$ \\
\hline $0-1$ & $62(65.3)$ & $12(27.9)$ & \\
\hline$\|-\mid\|$ & $33(34.7)$ & $31(72.1)$ & \\
\hline Histopathology $(\mathrm{N}=129)$ & & & 0.335 \\
\hline Well differentiation & $5(5.8)$ & $1(2.3)$ & \\
\hline Moderate differentiation & $74(86.0)$ & $35(81.4)$ & \\
\hline Poor differentiation & $7(8.1)$ & $7(16.3)$ & \\
\hline LNH & $8.3(7.3-9.4)$ & $10.0(8.6-11.5)$ & 0.051 \\
\hline LVI & & & 0.428 \\
\hline Positive & $4(4.2)$ & $4(9.3)$ & \\
\hline Negative & $91(95.8)$ & $39(90.7)$ & \\
\hline PNI & & & $<0.001$ \\
\hline Positve & $5(5.3)$ & $12(27.9)$ & \\
\hline Negative & $90(94.7)$ & $31(72.1)$ & \\
\hline Tumor deposits & & & 0.148 \\
\hline Positive & $11(11.6)$ & $9(20.9)$ & \\
\hline Negative & $84(88.4)$ & $34(79.1)$ & \\
\hline TRG & & & 0.001 \\
\hline $0-1$ & $64(67.4)$ & $16(37.2)$ & \\
\hline $2-3$ & $31(32.6)$ & $27(62.8)$ & \\
\hline CEA [median $(\mathrm{Cl})]$ & $2.6(2.4-2.9)$ & $5.3(3.9-7.1)$ & 0.002 \\
\hline FARI, \% [median(CI)] & $7.0(6.8-7.3)$ & $9.3(8.7-9.9)$ & $<0.001$ \\
\hline PNI [median $(\mathrm{Cl})]$ & $47.4(46.8-48.0)$ & $42.5(41.4-43.5)$ & $<0.001$ \\
\hline
\end{tabular}

$C /$ confidence interval, $L N H$ lymph node harvest, $L V I$ lymphovascular invasion, $P N /$ perineural invasion, TRG tumor regression grade, CEA carcinoembryonic antigen, FARI Fibrinogen-Albumin Ratio Index, PNI prognostic nutritional index

logistic regression analysis, cT4, mid-low tumor site, low PNI, and high CFP were significantly associated with a poor response, while high CEA and high FARI were not (Fig. 5b). In multivariate logistic regression analysis, cT stage (cT4 vs cT2-3, HR=2.837, $\mathrm{p}=0.040$ ), tumor site (lower vs upper, $\mathrm{HR}=7.683, \mathrm{p}=0.004$; middle vs upper, $H R=3.562, \mathrm{p}=0.058$ ) and CFP (High vs Low, $\mathrm{HR}=3.693, \mathrm{p}=0.002$ ) remained significantly associated with TRG. Detailed data are shown in Additional file 1: Table S2. According to the independent risk factors of tumor response that derived from the multivariate logistic regression analysis, we established two nomograms to predict the risk of poor response, one containing CFP, tumor site, cT stage and one only containing tumor site and CT stage. The AUC of the nomogram with CFP (0.717) was better than that without CFP (0.656) $(\mathrm{p}<0.05)$. In addition, the calibration curve of the nomogram with CFP was closer to the ideal curve than that without CFP (Fig. 6c, d).

\section{Discussion}

Rectal cancer is considered a complex disease caused by the interaction of genetic and environmental factors, which also leads to its heterogeneous nature [10]. Although the application of NCRT could shrink the tumor, achieve the objective of downstaging, and reduce the difficulty of surgery and local recurrence rate, the survival of patients is still far from satisfactory. Currently, the high-risk pathological factors for poor prognosis of rectal cancer include poor differentiation, the presence of LVI, perineural invasion, and positive circumferential resection margins. However, these indicators are only available after surgery, limiting their prognostic role in preoperative evaluation. Moreover, the current definition of high-risk factors is clearly inadequate since many patients with high-risk parameters do not have systemic recurrence, while some patients are deemed to be lowrisk do. Therefore, the identification of a novel biomarker that could predict prognosis and tumor response is vital. Recently, studies have shown that CEA [7], FARI [20], and PNI [21] are practical predictors of survival and tumor response in LARC patients who underwent radical surgery after NCRT. Hence, we verified the prognostic role of these parameters and established a CFP scoring system. Our study is the first to evaluate the prognostic role of the CFP scoring system in LARC patients, and CFP showed great predictive ability in both survival and tumor response.

Cancer-related inflammation is a defensive response elicited by the body against the tumor, and there is growing evidence that the systemic inflammatory response plays a critical role in the development and progression of malignancy [10]. Combinations of leukocyte-based inflammation markers, such as the neutrophil to lymphocyte ratio, lymphocyte to monocyte ratio, platelet to lymphocyte ratio, and systemic immune-inflammation, have also been reported to be significantly associated with the prognosis of malignant tumors [22-24]. However, NCRT may reduce the total circulating leukocytes and interfere with the inflammatory response of the host, limiting the application of leukocyte-based inflammation biomarkers to predict the prognosis of LARC patients who underwent NCRT. Wang et al. [17] found that both neutrophil-to-lymphocyte, lymphocyte-to-monocyte, 

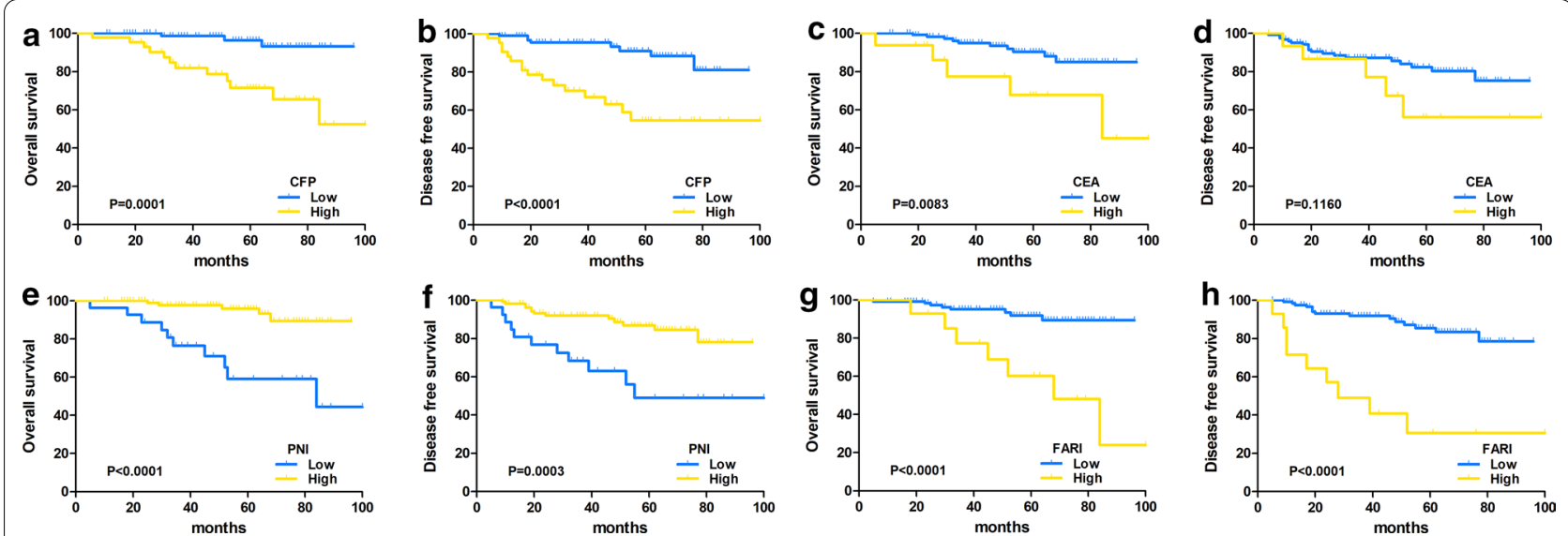

Fig. 4 Comparison of OS and DFS between different CEA, FARI, PNI and CFP groups in LARC patients. a Kaplan-Meier analysis for OS rate between different CFP groups in LARC patients $(p=0.0001)$. b Kaplan-Meier analysis for the DFS rate between different CFP groups in LARC patients $(p<0.0001)$. $\mathbf{c}$ Kaplan-Meier analysis for OS rate between different CEA groups in LARC patients $(p=0.0083)$. $\mathbf{d}$ Kaplan-Meier analysis for the DFS rate between different CEA groups in LARC patients $(p=0.1160)$. e Kaplan-Meier analysis for OS rate between different PNI groups in LARC patients $(p<0.0001)$. $\mathbf{f}$ Kaplan-Meier analysis for the DFS rate between different PNI groups in LARC patients $(p=0.0003)$. $\mathbf{g}$ Kaplan-Meier analysis for OS rate between different FARI groups in LARC patients $(p<0.0001)$. $\mathbf{h}$ Kaplan-Meier analysis for the DFS rate between different FARI groups in LARC patients $(p<0.0001)$.

Table 3 Univariable and multivariable analyses to determine independent predictors of DFS and OS in LARC patients

\begin{tabular}{|c|c|c|c|c|c|c|c|c|}
\hline & \multirow{2}{*}{$\begin{array}{l}\text { OS } \\
\text { Univariate }\end{array}$} & \multicolumn{2}{|l|}{$P$ value } & \multirow[t]{2}{*}{$P$ value } & \multirow{2}{*}{$\begin{array}{l}\text { DFS } \\
\text { Univariate }\end{array}$} & \multirow[t]{2}{*}{$P$ value } & & \multirow[t]{2}{*}{$P$ value } \\
\hline & & & Multivariate & & & & Multivariate & \\
\hline & HR (95\%Cl) & & HR $(95 \% C l)$ & & HR (95\%Cl) & & HR (95\%Cl) & \\
\hline $\begin{array}{l}\text { Gender (male vs } \\
\text { female) }\end{array}$ & $0.289(0.064-1.301)$ & 0.106 & - & - & $0.594(0.221-1.598)$ & 0.303 & - & - \\
\hline Age, years & $1.019(0.976-1.064)$ & 0.383 & - & - & $1.006(0.974-1.039)$ & 0.725 & - & - \\
\hline Tumor site & - & 0.736 & - & - & - & 0.272 & - & - \\
\hline Low vs Upper & $0.529(0.107-2.625)$ & 0.436 & - & - & $0.447(0.144-1.398)$ & 0.164 & - & - \\
\hline Middle vs Upper & $0.757(0.205-2.802)$ & 0.677 & - & - & $0.478(0.178-1.279)$ & 0.141 & - & - \\
\hline $\begin{array}{l}\text { Tumor size (>5 } \\
\text { vs } \leq 5)\end{array}$ & $3.119(0.990-9.822)$ & 0.052 & - & - & 3.819 (1.507-9.679) & 0.005 & - & - \\
\hline ypTNM (0-I vs II-III) & $5.357(1.505-19.070)$ & 0.010 & - & - & $\begin{array}{l}10.853(3.224- \\
36.529)\end{array}$ & $<0.001$ & $4.785(1.274-17.966)$ & 0.020 \\
\hline LNH & $0.999(0.901-1.107)$ & 0.980 & - & - & $1.035(0.957-1.119)$ & 0.385 & - & - \\
\hline LVI (+ vs -) & $\begin{array}{l}11.976(3.712- \\
38.637)\end{array}$ & $<0.001$ & $7.019(2.117-23.267)$ & 0.001 & $6.990(2.311-21.140)$ & 0.001 & - & - \\
\hline $\begin{array}{l}\text { Perineural invasion } \\
(+\mathrm{vs}-)\end{array}$ & $6.505(2.340-18.087)$ & $<0.001$ & - & - & $5.077(2.210-11.661)$ & $<0.001$ & $4.904(1.475-16.301)$ & 0.009 \\
\hline $\begin{array}{l}\text { Tumor deposit } \\
\text { (+vs }-)\end{array}$ & $4.476(1.569-12.768)$ & 0.005 & - & - & $6.867(3.061-15.406)$ & $<0.001$ & $7.932(2.731-23.038)$ & $<0.001$ \\
\hline CEA (High vs Low) & $3.891(1.316-11.507)$ & 0.014 & - & - & $2.160(0.806-5.791)$ & 0.126 & - & - \\
\hline FARI (High vs Low) & $6.495(2.349-17.959)$ & $<0.001$ & - & - & $7.274(3.171-16.686)$ & $<0.001$ & $3.642(1.316-10.081)$ & 0.013 \\
\hline PNI (Low vs High) & $7.764(2.643-22.810)$ & $<0.001$ & - & - & $3.922(1.753-8.773)$ & 0.001 & - & - \\
\hline CFP (High vs Low) & $8.117(2.288-28.789)$ & 0.001 & 6.606 (1.786-23.705) & 0.005 & $4.994(2.135-11.682)$ & $<0.001$ & $6.635(1.934-22.767)$ & 0.003 \\
\hline
\end{tabular}

$H R$ hazard ratio, $C l$ cofidence interval, $L N H$ lymph node harvest, $L V I$ lymphovascular invasion, $P N /$ perineural invasion, CEA carcinoembryonic antigen, $P N /$ prognostic nutritional index, FARI Fibrinogen-Albumin Ratio Index, CFP CEA-FARI-PNI score

platelet-to-lymphocyte and systemic immune-inflammation index (derived from lymphocyte, neutrophil and platelet counts) failed to show an independent prognostic value in patients undertook NCRT. Further, our previous findings were consistent with this point of view [20]. The CFP scoring system is a combination of tumor markers 

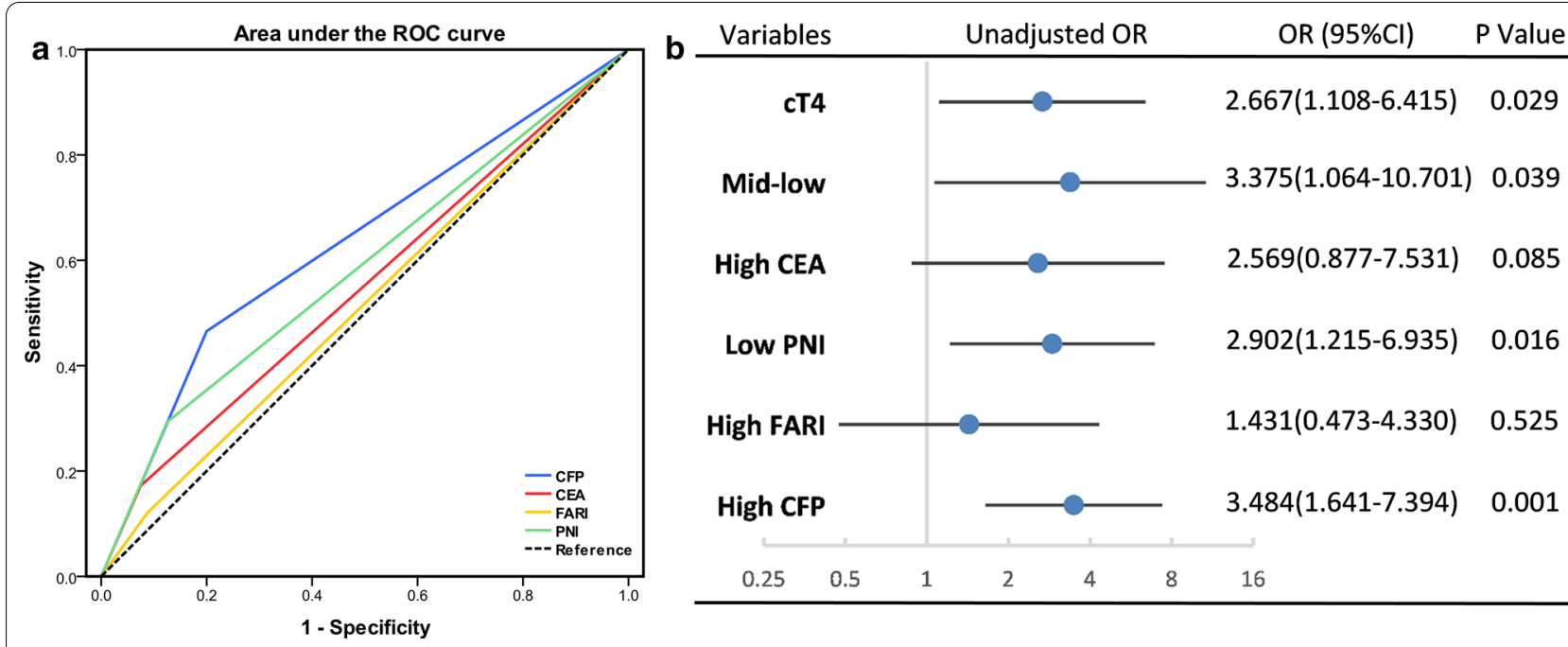

Fig. 5 ROC curve and forest plot. a ROC curve of CEA, FARI, PNI, and CFP predicts poor response. b Forest plot of unadjusted logistic regression to assess the discrimination ability of CEA, FARI, PNI, and CFP for tumor response.

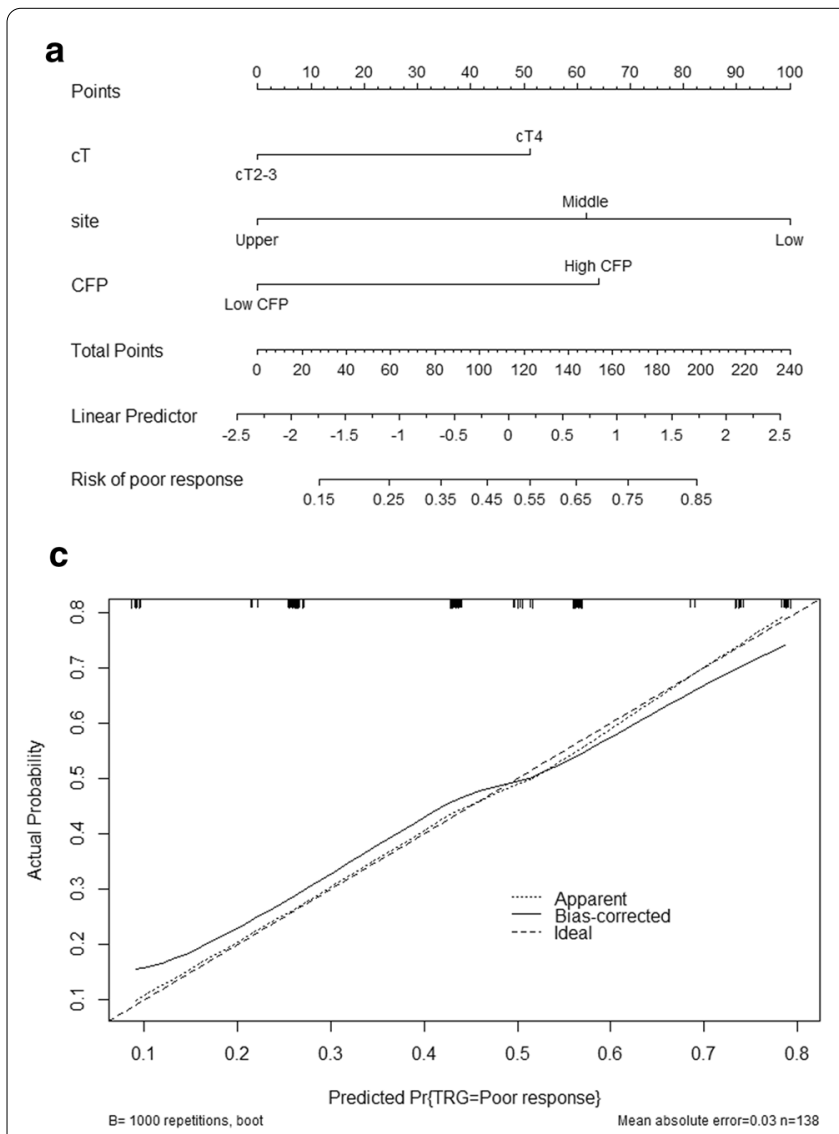

\section{b}

Points

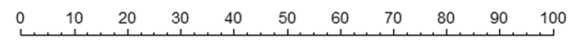

cT

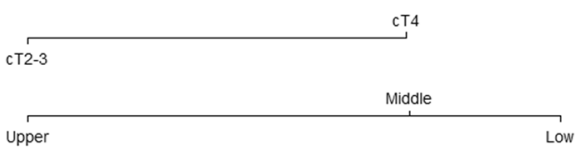

site

Total Points

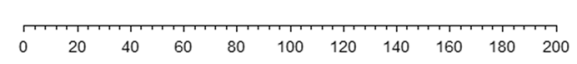

Linear Predictor

Risk of poor response

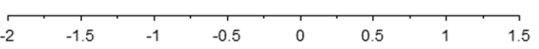

d

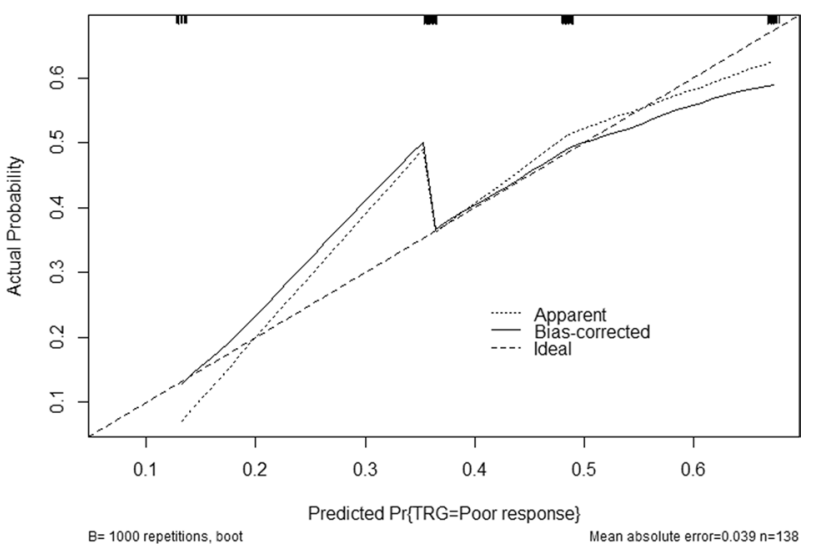

Fig. 6 Predicted nomogram and calibration of TRG. Predicted nomogram and calibration of TRG were established by significant factors. a Nomogram with CFP score; $\mathbf{b}$ without CFP score; $\mathbf{c}$ Calibration with CFP score; $\mathbf{d}$ without CDP score. The diagonal black dotted line represents a perfect prediction model. The solid black line represents the performance of the nomogram, and a closer fit to the diagonal black dotted line represents a better prediction. 
(CEA), inflammatory factors (lymphocytes and fibrinogen), and nutritional factors (albumin). We found that the CFP score based on CEA, FARI and PNI was superior to a single biomarker for precisely predicting the cancer burden and prognosis of the disease for the following reasons. First, lymphocytes, especially $\mathrm{CD}^{+}$and $\mathrm{CD} 8^{+} \mathrm{T}$ cells, migrate into the tumor microenvironment of LARC patients and play an essential antitumor role. EL Sissy et al. [25] found that the presence of $\mathrm{CD}^{+}$and $\mathrm{CD} 8^{+}$ $\mathrm{T}$ cells was correlated with survival in LARC patients. Second, the level of circulating fibrinogen is increased by interleukin- 6 secreted by tumor cells, and fibrinogen has been found to interact with several growth factors to induce tumor seeding and promote the invasion of tumor cells, leading to a poor prognosis [26]. Third, poor nutritional status is reflected by circulating albumin, which promotes IL-1, IL-6, TNF- $\alpha$, and acute-phase reactant release, increasing the morbidity and mortality of patients [27].

In our study, CEA, fibrinogen, albumin, and the total lymphocyte count were routine indicators examined before curative surgery, as well as FARI and PNI were the combinations of some of these indicators, making these biomarkers inexpensive and clinically practical. We found that high FARI, low PNI, and a CFP score of 1 were significantly associated with poor DFS and OS. CEA is also closely related to OS, but for DFS, there is only a tendency for a high CEA level to predict a poor DFS. The time-dependent ROC curve indicated that CFP has stable predictive performance in both OS and DFS for each time period and is an independent prognostic risk factor for both OS $(\mathrm{HR}=6.606, \mathrm{p}=0.005)$ and DFS $(\mathrm{HR}=6.635$, $\mathrm{p}=0.003$ ), suggesting that the novel CFP score was an appropriate biomarker for forecasting survival in LARC patients who underwent TME following NCRT. Recently, some researchers found that tumor budding, inflammatory infiltration and redox status could predict the prognosis of colorectal cancer and leads to new prognostic subgroups [28-30]. Some researchers found that clinicgenetic profile, which contain a complete summary of the patient status, and Raman-enhanced spectroscopy probe providing new possibilities in personalized medicine and prognostic views in cancer patient [31, 32]. In our future study, we will combine these promising findings with CFP to predict prognosis and tumor response among LARC patients.

The TRG scoring system provides a clinically useful indicator of tumor response to chemoradiotherapy and guides subsequent adjuvant treatment. Patients who achieve PCR do not need adjuvant therapy. Various TRG scoring systems exist, including quantitative and semiquantitative scoring systems, to grade the ratio between fiber and residual tumor cells [33-35].
By comparing the four most commonly used TRG systems, Trakarnsanga et al. [36] found that AJCC-TRG was the most accurate. These TRG systems can indeed predict improved DFS and OS [37], but TRG can only be obtained after surgical resection and cannot be used for prediction before surgery. Currently, rectal cancer patients who achieve a clinical complete response can use a watch and wait approach to avoid a series of complications and the associated risk of perioperative death caused by the TME procedure. Post-NCRT examinations such as digital rectal examination, endorectal ultrasonography, and magnetic resonance imaging (MRI) were used to determine the clinical complete response of LARC patients [38]. However, Liu et al. [39] performed the aforementioned examinations on 124 rectal cancer patients who underwent NCRT and found that although mucosal integrity, endorectal ultrasound, and MRI had a high specificity $(94.23,93.90$, and 93.27\%, respectively) for predicting complete response, their sensitivity was only $25 \%$. In addition, blood-based biomarkers such as circulating tumor DNA [40] and the modified Glasgow prognostic score [13] were associated with tumor response. However, these indicators were not routinely tested during treatment, possibly limiting their utility. Therefore, we further explored the association between CFP and NCRT outcomes, and our findings showed that the AUC (0.633) of CFP was superior to CEA (0.549), FARI (0.517), and PNI (0.584). Multivariate analysis indicated that a high CFP score $(\mathrm{HR}=3.693, \mathrm{p}=0.002)$ was an independent risk factor for poor tumor response (TRG2-3). We combined the clinical $\mathrm{T}$ stage, tumor site, and CFP to establish a nomogram that predicted the probability of poor response, and the AUC was 0.717, which was better than the AUC (0.656) without CFP $(p<0.05)$, suggesting that CFP is a reliable predictor for TRG.

However, some limitations exist in this study. First, this is a retrospective study, so some selection bias inevitably exists. Second, the sample size of this study is relatively small, reflecting the difficulties of subgroup analysis, and external validation of the existing results is lacking. In the future, more patients should be included, and the follow-up time should be extended to further verify these findings. In summary, this study is the first to construct a CEA-FARIPNI score and to investigate the predictive role of survival and chemoradiotherapy outcome in CEA, FARI, PNI, and CFP scores. The CFP score is a biomarker routinely measured in clinical practice and is an available and promising biomarker for predicting not only prognosis but also chemoradiotherapy outcome in LARC patients who underwent radical surgery after NCRT. 


\section{Conclusion}

In summary, our findings indicate that the CFP score is an effective and independent prognostic factor of OS and DFS for patients with LARC undergoing NCRT, and it could also effectively predict the tumor response.

\section{Supplementary Information}

The online version contains supplementary material available at https://doi. org/10.1186/s12935-021-01903-1.

Additional file 1: Figure S1. Flowchart of eligible cases selection.

Additional file 2: Table S1. AUC values of CEA, FARI, PNI and CFP.

Additional file 3: Table S2. Multivariate logistic regression analysis for TRG in LARC patients.

\section{Acknowledgements}

Not applicable.

\section{Authors' contributions}

SL and ZL collected and analyzed data, and wrote the manuscript. FL, BW, YM and JW contributed to data collection. YW and HW contributed to follow-up. $X Z$ and HW provided intellectual contribution. HW, XZ and WF supervised the project, discussed data analysis, and reviewed the manuscript.

\section{Funding}

This work was supported by grants from the National Natural Science Foundation of China (Grant Nos. 91959110 and 81972702), the Natural Science Foundation of Beijing (Grant No.7204324) and the National multidisciplinary cooperative diagnosis and treatment capacity building project for major diseases: comprehensive diagnosis and treatment of gastrointestinal tumors.

\section{Availability of data and materials}

The data that support the findings of this study are available on request from the corresponding author. The data are not publicly available due to privacy or ethical restrictions.

\section{Declarations}

\section{Ethics approval and consent to participate}

Ethical approval was obtained from the Ethics Committee of Peking University Third Hospital, and this study adhered to the tenets of the Declaration of Helsinki.

\section{Consent for publication \\ All authors agree to publish.}

\section{Competing interests}

The authors declare no competing interest.

Received: 22 November 2020 Accepted: 30 March 2021 Published online: 13 April 2021

\section{References}

1. Bray F, Ferlay J, Soerjomataram I, et al. Global cancer statistics 2018: GLOBOCAN estimates of incidence and mortality worldwide for 36 cancers in 185 countries. CA Cancer J Clin. 2018;68(6):394-424. https://doi.org/10. 3322/caac.21492.

2. Siegel RL, Miller KD, Goding Sauer A, et al. Colorectal cancer statistics, 2020. CA Cancer J Clin. 2020;70(3):145-64. https://doi.org/10.3322/caac. 21601.

3. Collette $\mathrm{L}$, Bosset JF, den Dulk M, et al. Patients with curative resection of CT3-4 rectal cancer after preoperative radiotherapy or radiochemotherapy: does anybody benefit from adjuvant fluorouracil-based chemotherapy? A trial of the European Organisation for Research and Treatment of Cancer Radiation Oncology Group. J Clin Oncol. 2007;25(28):4379-86. https://doi.org/10.1200/JCO.2007.11.9685.

4. Fleshman J, Branda ME, Sargent DJ, et al. Disease-free survival and local recurrence for laparoscopic resection compared with open resection of stage ii to iii rectal cancer: follow-up results of the ACOSOG Z6051 randomized controlled trial. Ann Surg. 2019;269(4):589-95. https://doi.org/10. 1097/SLA.0000000000003002.

5. Voss RK, Lin JC, Roper MT, et al. Adjuvant chemotherapy does not improve recurrence-free survival in patients with stage 2 or stage 3 rectal cancer after neoadjuvant chemoradiotherapy and total mesorectal excision. Dis Colon Rectum. 2020;63(4):427-40. https://doi.org/10.1097/DCR. 0000000000001558.

6. Nakamura Y, Shida D, Tanabe T, et al. Prognostic impact of preoperatively elevated and postoperatively normalized carcinoembryonic antigen levels following curative resection of stage I-III rectal cancer. Cancer Med. 2020;9(2):653-62. https://doi.org/10.1002/cam4.2758.

7. Perez RO, Sao Juliao GP, Habr-Gama A, et al. The role of carcinoembriogenic antigen in predicting response and survival to neoadjuvant chemoradiotherapy for distal rectal cancer. Dis Colon Rectum. 2009;52(6):1137-43. https://doi.org/10.1007/DCR.0b013e31819ef76b.

8. Jang NY, Kang SB, Kim DW, et al. The role of carcinoembryonic antigen after neoadjuvant chemoradiotherapy in patients with rectal cancer. Dis Colon Rectum. 2011;54(2):245-52. https://doi.org/10.1007/DCR.0b013 e3181fcee68.

9. Candido J, Hagemann T. Cancer-related inflammation. J Clin Immunol. 2013;33(Suppl 1):S79-84. https://doi.org/10.1007/s10875-012-9847-0.

10. Kuipers EJ, Grady WM, Lieberman D, et al. Colorectal cancer. Nat Rev Dis Primers. 2015;1:15065. https://doi.org/10.1038/nrdp.2015.65.

11. Shawki S, Ashburn J, Signs SA, et al. Colon cancer: inflammation-associated cancer. Surg Oncol Clin N Am. 2018;27(2):269-87. https://doi.org/10. 1016/j.soc.2017.11.003.

12. Mantovani A, Allavena P, Sica A, et al. Cancer-related inflammation. Nature. 2008;454(7203):436-44. https://doi.org/10.1038/nature07205.

13. Dreyer SB, Powell AG, McSorley ST, et al. The pretreatment systemic inflammatory response is an important determinant of poor pathologic response for patients undergoing neoadjuvant therapy for rectal cancer. Ann Surg Oncol. 2017;24(5):1295-303. https://doi.org/10.1245/ s10434-016-5684-3.

14. Chandra RK. Nutrition and the immune system: an introduction. Am J Clin Nutr. 1997;66(2):460S-S463. https://doi.org/10.1093/ajcn/66.2.460S.

15. Jager-Wittenaar $H$, Dijkstra PU, Vissink A, et al. Malnutrition and quality of life in patients treated for oral or oropharyngeal cancer. Head Neck. 2011;33(4):490-6. https://doi.org/10.1002/hed.21473.

16. Chen QG, Zhang L, Sun F, et al. Elevated FPR confers to radiochemoresistance and predicts clinical efficacy and outcome of metastatic colorectal cancer patients. Aging (Albany NY). 2019;11(6):1716-32. https://doi.org/ 10.18632/aging.101864.

17. Wang YY, Liu ZZ, Xu D, et al. Fibrinogen-Albumin Ratio Index (FARI): a more promising inflammation-based prognostic marker for patients undergoing hepatectomy for colorectal liver metastases. Ann Surg Oncol. 2019;26(11):3682-92. https://doi.org/10.1245/s10434-019-07586-3.

18. Tan Z, Zhang M, Han Q, et al. A novel blood tool of cancer prognosis in esophageal squamous cell carcinoma: the Fibrinogen/Albumin Ratio. J Cancer. 2017;8(6):1025-9. https://doi.org/10.7150/jca.16491.

19. Zhang J, Ruan J, Wang W, et al. Prognostic value of the combination of CEA and fibrinogen/albumin ratio in resectable gastric cancer. Cancer Manag Res. 2020;12:2767-75. https://doi.org/10.2147/CMAR.S246566.

20. Lu S, Liu Z, Zhou X, et al. Preoperative Fibrinogen-Albumin Ratio Index (FARI) is a reliable prognosis and chemoradiotherapy sensitivity predictor in locally advanced rectal cancer patients undergoing radical surgery following neoadjuvant chemoradiotherapy. Cancer Manag Res. 2020;12:8555-68. https://doi.org/10.2147/CMAR.S273065.

21. Okugawa $Y$, Toiyama $Y$, Oki S, et al. Feasibility of assessing prognostic nutrition index in patients with rectal cancer who receive preoperative chemoradiotherapy. JPEN J Parenter Enteral Nutr. 2018;42(6):998-1007. https://doi.org/10.1002/jpen.1041.

22. Goto W, Kashiwagi S, Asano Y, et al. Predictive value of lymphocyte-tomonocyte ratio in the preoperative setting for progression of patients with breast cancer. BMC Cancer. 2018;18(1):1137. https://doi.org/10.1186/ s12885-018-5051-9. 
23. Yang $L, H e W$, Kong $P$, et al. Clinical baseline and prognostic difference of platelet lymphocyte ratio $(P L R)$ in right-sided and let-sided colon cancers. BMC Cancer. 2017;17(1):873. https://doi.org/10.1186/s12885-017-3862-8.

24. Chen JH, Zhai ET, Yuan YJ, et al. Systemic immune-inflammation index for predicting prognosis of colorectal cancer. World J Gastroenterol. 2017;23(34):6261-72. https://doi.org/10.3748/wjg.v23.i34.6261.

25. El Sissy C, Kirilovsky A, Van den Eynde M, et al. A diagnostic biopsyadapted immunoscore predicts response to neoadjuvant treatment and selects patients with rectal cancer eligible for a watch-and-wait strategy. Clin Cancer Res. 2020;26(19):5198-207. https://doi.org/10.1158/10780432.CCR-20-0337.

26. Shu YJ, Weng H, Bao RF, et al. Clinical and prognostic significance of preoperative plasma hyperfibrinogenemia in gallbladder cancer patients following surgical resection: a retrospective and in vitro study. BMC Cancer. 2014;14:566. https://doi.org/10.1186/1471-2407-14-566.

27. Gupta D, Lis CG. Pretreatment serum albumin as a predictor of cancer survival: a systematic review of the epidemiological literature. Nutr J. 2010;9:69. https://doi.org/10.1186/1475-2891-9-69.

28. Lang-Schwarz C, Melcher B, Haumaier F, et al. Budding and tumor-infiltrating lymphocytes - combination of both parameters predicts survival in colorectal cancer and leads to new prognostic subgroups. Hum Pathol. 2018:79:160-7. https://doi.org/10.1016/j.humpath.2018.05.010.

29. Zinczuk J, Maciejczyk M, Zareba K, et al. Antioxidant barrier, redox status, and oxidative damage to biomolecules in patients with colorectal cancer. Can malondialdehyde and catalase be markers of colorectal cancer advancement? Biomolecules. 2019. https://doi.org/10.3390/biom9 100637.

30. Zinczuk J, Maciejczyk M, Zareba K, et al. Pro-oxidant enzymes, redox balance and oxidative damage to proteins, lipids and DNA in colorectal cancer tissue. Is oxidative stress dependent on tumour budding and inflammatory infiltration? Cancers. 2020. https://doi.org/10.3390/cance rs12061636.

31. Agsalda-Garcia M, Shieh T, Souza R, et al. Raman-Enhanced Spectroscopy (RESpect) probe for childhood non-hodgkin lymphoma. Sci Med J. 2020;2(1):1-7. https://doi.org/10.28991/SciMedJ-2020-0201-1.

32. Kosvyra A, Maramis C, Chouvarda I. Developing an integrated genomic profile for cancer patients with the use of NGS data. Emerg Sci J. 2019;3(3):157-67. https://doi.org/10.28991/esj-2019-01178.

33. Mandard AM, Dalibard F, Mandard JC, et al. Pathologic assessment of tumor regression after preoperative chemoradiotherapy of esophageal carcinoma. Clinicopathol Correlat Cancer. 1994;73(11):2680-6. https://doi. org/10.1002/1097-0142(19940601)73:11\%3c2680::aid-cncr2820731105\% 3e3.0.co;2-c

34. Quah HM, Chou JF, Gonen M, et al. Pathologic stage is most prognostic of disease-free survival in locally advanced rectal cancer patients after preoperative chemoradiation. Cancer. 2008;113(1):57-64. https://doi.org/ 10.1002/cncr.23516.

35. Jager T, Neureiter D, Urbas R, et al. Applicability of American Joint Committee on cancer and college of american pathologists regression grading system in rectal cancer. Dis Colon Rectum. 2017;60(8):815-26. https:// doi.org/10.1097/DCR.0000000000000806.

36. Trakarnsanga A, Gonen M, Shia J, et al. Comparison of tumor regression grade systems for locally advanced rectal cancer after multimodality treatment. J Natl Cancer Inst. 2014. https://doi.org/10.1093/jnci/dju248.

37. Huh JW, Kim HC, Kim SH, et al. Tumor regression grade as a clinically useful outcome predictor in patients with rectal cancer after preoperative chemoradiotherapy. Surgery. 2019;165(3):579-85. https://doi.org/10. 1016/j.surg.2018.08.026.

38. Habr-Gama A, Perez RO, Wynn G, et al. Complete clinical response after neoadjuvant chemoradiation therapy for distal rectal cancer: characterization of clinical and endoscopic findings for standardization. Dis Colon Rectum. 2010;53(12):1692-8. https://doi.org/10.1007/DCR.0b013e3181 f42b89.

39. Liu S, Zhong GX, Zhou WX, et al. Can endorectal ultrasound, mri, and mucosa integrity accurately predict the complete response for midlow rectal cancer after preoperative chemoradiation? A prospective observational study from a single medical center. Dis Colon Rectum. 2018;61(8):903-10. https://doi.org/10.1097/DCR.0000000000001135.

40. Murahashi S, Akiyoshi T, Sano T, et al. Serial circulating tumour DNA analysis for locally advanced rectal cancer treated with preoperative therapy: prediction of pathological response and postoperative recurrence. $\mathrm{Br}$ J Cancer. 2020;123(5):803-10. https://doi.org/10.1038/s41416-020-0941-4.

\section{Publisher's Note}

Springer Nature remains neutral with regard to jurisdictional claims in published maps and institutional affiliations.
Ready to submit your research? Choose BMC and benefit from:

- fast, convenient online submission

- thorough peer review by experienced researchers in your field

- rapid publication on acceptance

- support for research data, including large and complex data types

- gold Open Access which fosters wider collaboration and increased citations

- maximum visibility for your research: over $100 \mathrm{M}$ website views per year

At BMC, research is always in progress.

Learn more biomedcentral.com/submissions 